\title{
Polycyclic Aromatic Hydrocarbons and the Risk of Kidney Stones in US Adults: An Exposure- Response Analysis of NHANES 2007-20I2
}

\author{
Si Sun ${ }^{1, *}$ \\ Weipu Mao ${ }^{\mathrm{I}-3, *}$ \\ Shuchun Tao',* \\ Xiangyu Zou ${ }^{4}$ \\ Shengwei Tian' \\ Siwei Qian' \\ Chi Yao' \\ Guangyuan Zhang ${ }^{1,2}$ \\ Ming Chen ${ }^{1-3}$
}

'Department of Urology, Zhongda Hospital, Southeast University, Nanjing, 210009, People's Republic of China;

${ }^{2}$ Surgical Research Center, Institute of Urology, Southeast University Medical School, Nanjing, 210009, People's Republic of China; ${ }^{3}$ Department of Urology, Nanjing Lishui District People's Hospital, Zhongda Hospital Lishui Branch, Southeast University, Nanjing, 2 I I200, People's Republic of China; ${ }^{4}$ Department of Urology, Shanghai Children's Medical Center, Shanghai Jiao Tong University School of Medicine, Shanghai, 200I27, People's Republic of China

*These authors contributed equally to this work

Correspondence: Ming Chen; Guangyuan Zhang

Department of Urology, Zhongda Hospital, Southeast University, No. 87 Dingjiaqiao, Hunan Road, Gulou District, Nanjing, 210009, People's Republic of China Tel +86-I 39 I3009977; +86-I 3951658634 Fax +86-I39 I3009977; +86-I395I658634 Email mingchenseu@।26.com; zgy0879@qq.com
Background: Polycyclic aromatic hydrocarbons (PAHs) exposure may cause various diseases. However, the association between PAHs exposure and kidney stones remains unclear. The purpose of this study was to examine the relationship between PAHs and the risk of kidney stones in the US population.

Methods: The study included a total of 30,442 individuals ( $\geq 20$ years) from the 2007-2012 National Health and Nutrition Examination Survey (NHANES). Nine urinary PAHs were included in this study. Logistic regression and dose-response curves were used to evaluate the association between PAHs and the risk of kidney stones.

Results: We selected 4385 participants. The dose-response curves showed a significant positive association between total PAHs, 2-hydroxynaphthalene, 1-hydroxyphenanthrene, 2-hydroxyphenanthrene, 9-hydroxyfluorene and the risk of kidney stones after adjusting for confounding factors. Compared with the low group, an increased risk of kidney stones was observed in the high group of total PAHs [OR (95\% CI), 1.32 (1.06-1.64), P=0.013], 2-hydroxynaphthalene [OR (95\% CI), 1.37 (1.10-1.71), $\mathrm{P}=0.005], 1$-hydroxyphenanthrene [OR (95\% CI), 1.24 (1.00-1.54), P=0.046] and 9-hydroxyfluorene [OR (95\% CI), 1.36 (1.09-1.70), $\mathrm{P}=0.007]$.

Conclusion: High levels of PAHs were positively associated with the risk of kidney stones in the US population.

Keywords: polycyclic aromatic hydrocarbons, kidney stones, NHANES, cross-sectional survey

\section{Introduction}

Kidney stones are a common and frequently occurring disease of the urinary system, which are caused by the abnormal accumulation of crystal substances in the kidney. ${ }^{1}$ In recent years, the prevalence of kidney stones is $12 \%$ and $5 \%$ in adult men and women in western countries, respectively, and the incidence rate has increased. ${ }^{2}$ Kidney stones can cause serious complications, pain, hematuria, infection, decreased kidney function, and even kidney failure. ${ }^{3,4}$ Dietary and lifestyle factors play an important role in the risk of kidney stones, especially high animal protein intake increases the risk of kidney stone formation. ${ }^{5}$

Polycyclic aromatic hydrocarbons (PAHs) are hydrocarbon compounds containing more than two fused aromatic rings. To date, it has been found that PAHs consist of more than 200 compounds, such as naphthalene, anthracene, phenanthrene and pyrene. ${ }^{6,7}$ PAHs are generated from incomplete combustion of organic 
polymer compounds, including coal, petrochemicals, rubber, plastics, wood and tobacco, etc. ${ }^{8}$ PAHs have been detected in the human production and living environment. ${ }^{9}$ They are a typical class of persistent pollutants that can be rapidly enriched in organisms and cause various hazards. ${ }^{10}$ PAHs can be absorbed into the body by lungs, gut and skin, and be metabolized and eliminated in the urine within a short period. ${ }^{11}$ Therefore, urinary PAHs concentrations are considered biomarkers of exposure to PAHs. $^{12}$

As a typical persistent organic pollutant, previous studies have shown that the main target organ of PAHs is the lung, but the liver, with its detoxification function, and the kidney, with its excretion function, may also be affected by PAHs exposure. ${ }^{13}$ Studies on the mechanism of damage caused by PAHs have shown that PAHs can enhance the body's oxidative stress and increase the body's reactive oxygen species. ${ }^{14-16}$ However, the association between PAHs and the risk of kidney stones remains unclear. We aim to investigate whether the level of PAHs in a representative population sample from the National Health and Nutrition Survey (NHANES) is associated with the risk of kidney stones.

\section{Patients and Methods}

\section{Patients Selection}

NHANES is a nationally representative research program that uses a complex stratified multistage sampling design to assess children and adults' health and nutritional status in the United States. The project has been conducted every two years since 1999, and data are collected through household interviews and health screenings. The NHANES Institutional Review Board approved the study protocol for the project, and all participants signed informed consent forms during the survey. The study included 30,442 individuals ( $\geq 20$ years) from the NHANES 2007-2008, 2009-2010, and 2011-2012 cycles. More details are available on the web (www.cdc.gov/nchs/ nhanes/).

The exclusion criteria were as follows: (a) Patients who had not completed the survey (kidney stones KIQ026) (n=12,784); (b) unknown PAHs (n=12,439); (c) unknown family poverty ratio $(n=478)$; (d) unknown BMI $(n=51)$; (e) unknown creatinine $(\mathrm{n}=259)$; (f) unknown uric acid $(\mathrm{n}=2)$; (g) abnormal PAHs $(\mathrm{n}=44)$. After excluding all factors, 4385 participants were selected.

\section{Study Variables and Outcome}

Total PAHs are the main predictors in this study, including 1-hydroxynaphthalene, 2-hydroxynaphthalene, 3-hydroxyfluorene, 2-hydroxyfluorene, 3-hydroxyphenanthrene, 1-hydroxyphenanthrene, 2-hydroxyphenanthrene, 1-hydroxypyrene and 9-hydroxyfluorene. According to the website (wwwn.cdc.gov/nchs/data/nhanes/2011-2012/labmethods/ pah_g_met.pdf), urinary PAHs were measured by standard procedures.

Besides, we included other variables, such as gender, age, race, education level, marital status, family poverty ratio, body mass index (BMI), hypertension, diabetes, recreational activities. Based on the Chronic Kidney Disease Epidemiology Collaboration equation, we calculate the estimated glomerular filtration rate (eGFR) of all participants. $^{17}$

Male: $\operatorname{GFR}=141 \times \min (\mathrm{Scr} / 0.9,1)^{-0.411} \times \max (\mathrm{Scr} /$ $0.9,1)^{-1.209} \times 0.993^{\text {Age }} \times 1.159$ (if black).

Female: GFR $=141 \times \min (\mathrm{Scr} / 0.7,1)^{-0.329} \times \max$ $(\mathrm{Scr} / 0.7,1)^{-1.209} \times 0.993^{\text {Age }} \times 1.018 \times 1.159$ (if black) .

The endpoints of the study were the history of kidney stones. We extracted the relevant information from the questionnaire data file. In the questionnaire, participants responded to "Have you ever had kidney stones?". Those who answered "No" were considered to have no history of kidney stones.

\section{Statistical Analysis}

Continuous variables were expressed as mean \pm standard deviation, and t-tests for slope were used in the generalized linear model. Categorical variables were defined as $\mathrm{n}(\%)$ and analyzed by Chi-square analysis. Nine kinds of PAHs and total PAHs were divided into two groups. Binary logistic regression models were used to evaluate the corrected odds ratios (ORs) and 95\% confidence interval (CI) intervals for factors associated with kidney stones. In the extended model, based on the correlation of demographic characteristics with PAHs, we adjusted gender, age, race, education levels, marital status, family poverty, body mass index, hypertension, diabetes, recreational activities, blood urea nitrogen, uric acid, creatinine and eGFR of the participants.

The restricted cubic spline function is a powerful tool for describing dose-response relationships between continuous variables and outcomes. The restricted cubic spline function was applied to describe the dose-response relationship between PAHs and the risk of kidney stones and 


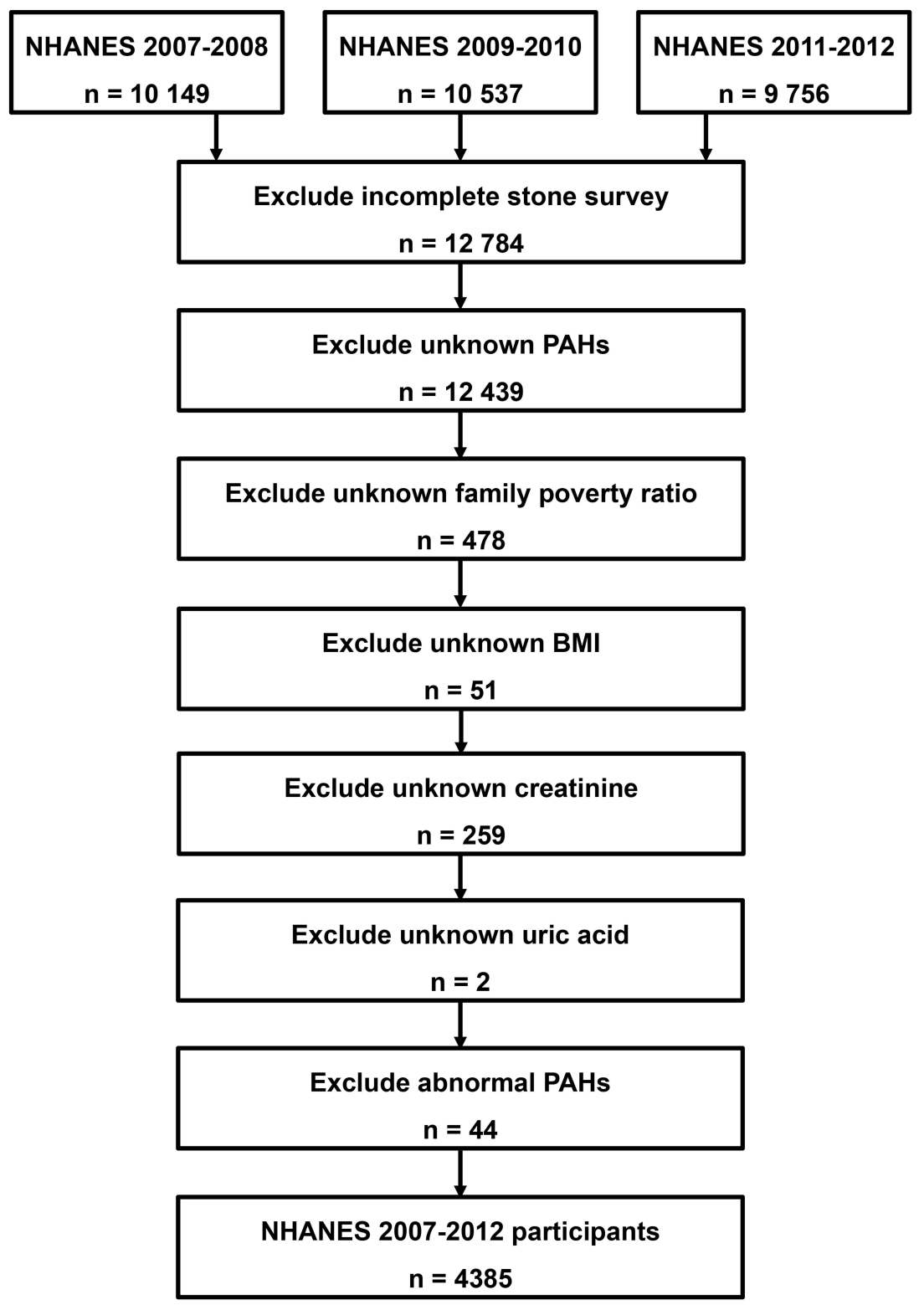

Figure I Schematic flow diagram of inclusion and exclusion criteria for our study cohort.

adjusted for the model variables. ${ }^{18}$ The study was analyzed using SPSS software (version 24.0) and RStudio software (version 1.2.5033), GraphPad software (version 8.0.2) and Origin 2018 software. $\mathrm{P}<0.05$ was considered statistically significant.

\section{Results}

\section{Participant Characteristics}

A total of 30,442 NHANES participants (2007-2012) were included. Of these participants, 5165 had complete data on urinary PAHs and stone investigations. According to the exclusion criteria, 4385 participants were finally included in the study (Figure 1). The characteristics and demographics of participants were shown in Table 1. Among them, 376 participants $(8.6 \%)$ had the stone formation, and 4009 participants $(91.4 \%)$ had no stone formation. The chi-square test indicated that several variables were significantly different between non-stone formers and stone formers, including gender, age, race, BMI, hypertension, diabetes, vigorous recreational activities, blood urea nitrogen, creatinine, Uric acid, eGFR and total PAHs. Participants with a history of kidney stones were mostly concentrated in men $(60.6 \%), \geq 50$ years $(58.8 \%)$, nonHispanic whites $(59.0 \%), \quad \mathrm{BMI} \geq 25 \quad \mathrm{~kg} / \mathrm{m}^{2} \quad(75.0 \%)$, 
Table I Baseline Characteristics of NHANES Participants Between 2007 and $2012^{\text {a }}$

\begin{tabular}{|c|c|c|c|c|}
\hline \multirow[t]{2}{*}{ Characteristic } & Total & $\begin{array}{c}\text { None-Stone } \\
\text { Formers }\end{array}$ & Stone Formers & \multirow[t]{2}{*}{$P$ value } \\
\hline & No. (\%) & No. (\%) & No. (\%) & \\
\hline Total participants & 4385 & 4009 (91.4) & $376(8.6)$ & \\
\hline $\begin{array}{l}\text { Gender } \\
\text { Male } \\
\text { Female }\end{array}$ & $\begin{array}{c}<0.001 \\
2196(50.1) \\
2189(49.9)\end{array}$ & $\begin{array}{l}\mid 968(49.1) \\
204 \mid(50.9)\end{array}$ & $\begin{array}{l}228(60.6) \\
148(39.4)\end{array}$ & \\
\hline $\begin{array}{l}\text { Age } \\
\text { Mean }(\mathrm{SD}) \\
<50 \text { years } \\
\geq 50 \text { years }\end{array}$ & $\begin{array}{c}<0.001 \\
48.57(17.70) \\
2314(52.8) \\
207 \mid(47.2)\end{array}$ & $\begin{array}{l}48.03(17.70) \\
2159(53.9) \\
1850(46.1)\end{array}$ & $\begin{array}{l}54.32(16.62) \\
155(4 \mid .2) \\
221(58.8)\end{array}$ & $<0.001$ \\
\hline $\begin{array}{l}\text { Race } \\
\text { Non-Hispanic white } \\
\text { Non-Hispanic black } \\
\text { Mexican American } \\
\text { Other Hispanic } \\
\text { Other }\end{array}$ & $\begin{array}{c}<0.001 \\
2009(45.8) \\
887(20.2) \\
649(14.8) \\
449(10.2) \\
391(8.0)\end{array}$ & $\begin{array}{l}1787(44.6) \\
844(21.1) \\
610(15.2) \\
399(10.0) \\
369(9.2)\end{array}$ & $\begin{array}{l}222(59.0) \\
43(11.4) \\
39(10.4) \\
50(13.3) \\
22(5.9)\end{array}$ & \\
\hline $\begin{array}{l}\text { Education } \\
\text { Less than high school } \\
\text { High school or equivalent } \\
\text { College or above } \\
\text { Other }\end{array}$ & $\begin{array}{c}0.516 \\
1130(25.8) \\
1010(23.0) \\
2240(51.1) \\
5(0.1)\end{array}$ & $\begin{array}{c}1040(25.9) \\
915(22.8) \\
2050(51.1) \\
4(0.1)\end{array}$ & $\begin{array}{c}90(23.9) \\
95(25.3) \\
190(50.5) \\
1(0.3)\end{array}$ & \\
\hline $\begin{array}{l}\text { Marital status } \\
\text { Married } \\
\text { Unmarried }\end{array}$ & $\begin{array}{c}0.133 \\
2275(51.9) \\
2110(48.1)\end{array}$ & $\begin{array}{l}2066(51.5) \\
1943(48.5)\end{array}$ & $\begin{array}{l}209(55.6) \\
167(44.4)\end{array}$ & \\
\hline $\begin{array}{l}\text { Family poverty ratio }(\%) \\
\qquad \begin{array}{l}<1.3 \% \\
\text { I.3-3.5\% } \\
\geq 3.5 \%\end{array}\end{array}$ & $\begin{array}{c}0.689 \\
|46|(33.3) \\
1572(35.8) \\
1352(30.8)\end{array}$ & $\begin{array}{l}1343(33.5) \\
1435(35.8) \\
|23|(30.7)\end{array}$ & $\begin{array}{l}118(31.4) \\
137(36.4) \\
121(32.2)\end{array}$ & \\
\hline $\begin{aligned} \text { BMI }\left(\mathrm{kg} / \mathrm{m}^{2}\right) \\
\text { Mean }(\mathrm{SD}) \\
<25 \mathrm{~kg} / \mathrm{m}^{2} \\
\geq 25 \mathrm{~kg} / \mathrm{m}^{2}\end{aligned}$ & $\begin{array}{c}0.025 \\
28.98(6.79) \\
1319(30.1) \\
3066(69.9)\end{array}$ & $\begin{array}{l}28.92(6.82) \\
1225(30.6) \\
2784(69.4)\end{array}$ & $\begin{array}{l}29.67(6.46) \\
94(25.0) \\
282(75.0)\end{array}$ & 0.040 \\
\hline $\begin{array}{l}\text { Hypertension } \\
\text { Yes } \\
\text { No/Unknown }\end{array}$ & $\begin{array}{c}<0.00 \mid \\
|49|(34.0) \\
2894(66.0)\end{array}$ & $\begin{array}{l}1326(33.1) \\
2683(66.9)\end{array}$ & $\begin{array}{l}165(43.9) \\
211(56.1)\end{array}$ & \\
\hline $\begin{array}{l}\text { Diabetes } \\
\text { Yes } \\
\text { No/Unknown }\end{array}$ & $\begin{array}{c}<0.001 \\
503(11.5) \\
3882(88.5)\end{array}$ & $\begin{array}{c}438(10.9) \\
3571(89.1)\end{array}$ & $\begin{array}{c}65(17.3) \\
311(82.7)\end{array}$ & \\
\hline $\begin{array}{l}\text { Vigorous recreational activities } \\
\text { Yes } \\
\text { No }\end{array}$ & $\begin{array}{c}0.002 \\
941(21.5) \\
3444(78.5)\end{array}$ & $\begin{array}{c}884(22.1) \\
3125(77.9)\end{array}$ & $\begin{array}{c}57(15.2) \\
319(84.8)\end{array}$ & \\
\hline $\begin{array}{l}\text { Moderate recreational activities } \\
\text { Yes } \\
\text { No }\end{array}$ & $\begin{array}{c}0.146 \\
1740(39.7) \\
2645(60.3)\end{array}$ & $\begin{array}{l}1604(40.0) \\
2405(60.0)\end{array}$ & $\begin{array}{l}136(36.2) \\
240(63.8)\end{array}$ & \\
\hline
\end{tabular}

(Continued) 
Table I (Continued).

\begin{tabular}{|c|c|c|c|c|}
\hline \multirow[t]{2}{*}{ Characteristic } & Total & $\begin{array}{l}\text { None-Stone } \\
\text { Formers }\end{array}$ & Stone Formers & \multirow[t]{2}{*}{$P$ value } \\
\hline & No. (\%) & No. (\%) & No. (\%) & \\
\hline Blood urea nitrogen, $\mathrm{mg} / \mathrm{dL}$ & $13.10(5.78)$ & $12.95(5.63)$ & $14.64(7.06)$ & $<0.001$ \\
\hline Creatinine, $\mathrm{mg} / \mathrm{dL}$ & $0.89(0.34)$ & $0.89(0.33)$ & $0.94(0.35)$ & 0.003 \\
\hline Uric acid, mg/dL & $5.50(1.45)$ & $5.48(1.44)$ & $5.73(1.57)$ & 0.001 \\
\hline eGFR & $94.99(23.6)$ & $95.60(23.57)$ & $88.4 \mathrm{I}(23.62)$ & $<0.001$ \\
\hline Total PAHs, ng/L & |47.4| (|88.43) & | $44.96(\mid 80.40)$ & $173.50(257.87)$ & 0.005 \\
\hline
\end{tabular}

${ }^{\text {a }}$ Notes: For categorical variables, $\mathrm{P}$ values were analyzed by chi-square tests. For continuous variables, the $t$-test for slope was used in generalized linear models. Abbreviations: BMI, body mass index; PAHs, polycyclic aromatic hydrocarbons; eGFR, estimated glomerular filtration rate.

hypertension-positive (43.9\%), diabetes-positive $(17.3 \%)$ and less vigorous recreational activities (84.8\%). Moreover, stone formers had higher blood urea nitrogen, creatinine, uric acid, total PAHs and lower eGFR than none-stone formers.

Based on the total PAHs levels, we divided all participants into two groups: $<79.66 \mathrm{ng} / \mathrm{L}$ (Low group) and $\geq 79.66 \mathrm{ng} / \mathrm{L}$ (High group). The clinical characteristics of participants in each group were shown in Table 2. Participants with higher total PAHs were concentrated in female, college or above, unmarried, family poverty ratio $<1.3 \%$, less recreational activities, kidney stones, higher creatinine, higher blood urea nitrogen, higher uric acid and lower eGFR. Moreover, similar results were observed in non-stone formers and stone formers (Tables S1 and $\underline{\mathrm{S} 2}$ ).

\section{Profiles of Urinary PAHs}

Spearman's rank correlation coefficients of PAHs, blood urea nitrogen, creatinine, uric acid and eGFR were shown in Figure S1. The distribution levels of PAHs in this study were shown in Table S3. We found that the concentrations of 1-hydroxynaphthalene and 2-hydroxynaphthalene were higher among the nine PAHs (Table S4).

\section{Urinary PAHs and Kidney Stone}

Logistic regression analysis was performed to further identify risk factors associated with the prevalence of kidney stones. In the extended model, after adjusting for gender, age, race, education levels, marital status, family poverty, body mass index, hypertension, diabetes, recreational activities, blood urea nitrogen, uric acid, creatinine and eGFR, we found a significant positive correlation between total PAHs, 2-hydroxyphenanthrene, 1-hydroxyphenanthrene, 9-hydroxyfluorene and the risk of kidney stones, and the adjusted ORs for the prevalence of kidney stones increased significantly with the increase of total PAHS [OR (95\% CI), 1.32 (1.06-1.64), $\mathrm{p}=0.013$ ], 2-hydroxynaphthalene [OR (95\% CI), 1.37 (1.10-1.71), $\mathrm{P}=0.005]$, 1-hydroxyphenanthrene [OR (95\% CI), $1.24(1.00-1.54), \mathrm{P}=0.046]$ and 9-hydroxyfluorene [OR $(95 \%$ CI $), 1.36$ (1.09-1.70), $\mathrm{P}=0.007]$ (Table 3, Figure 2). The nonlinear dose-response risk curve showed that the risk of kidney stones increased with the increase of total PAHs, 2-hydroxyphenanthrene, 1-hydroxyphenanthrene and 9-hydroxyfluorene. (Table 4, Figure 3).

\section{Discussion}

Kidney stones are a very common and recurring disease. It is considered to be caused by the abnormal accumulation of crystals in the kidneys. Among the various types of renal stones, those composed of Calcium oxalate $(\mathrm{CaOx})$ are the commonest. ${ }^{1}$ Although there are many treatment methods for stones, the incidence and recurrence rate of kidney stones are still high, which exerts serious effects on patients' lives. ${ }^{19}$ Therefore, exploring the risk factors of kidney stones is of great significance for reducing the social and economic burden and improving patients' postoperative recovery.

PAHs are hydrocarbons containing more than two benzene rings. They are widely distributed in our lives through the incomplete combustion of fossil fuels such as coal, petroleum and the release of tar, coal, coke, pitch, and cigarettes. ${ }^{20}$ Many studies have reported that PAHs may cause damage to the respiratory system, digestive system, circulatory system, and urinary system. ${ }^{21-23}$ Mallin et al found that PAHs exposure would increase 
Table 2 Characteristics of the Study Population by Categories of Total Polycyclic Aromatic Hydrocarbons (PAHs) Levels in NHANES 2007-2012 a

\begin{tabular}{|c|c|c|c|c|}
\hline \multirow[t]{2}{*}{ Characteristic } & \multirow{2}{*}{$\begin{array}{l}\text { Total } \\
\text { No. (\%) }\end{array}$} & \multicolumn{2}{|c|}{ Total PAHs Level } & \multirow[t]{2}{*}{$\mathbf{P}_{\text {value }}{ }^{\mathrm{a}}$} \\
\hline & & Low Group & High Group & \\
\hline Total participants & 4385 & $2193(50.0)$ & $2192(50.0)$ & \\
\hline Gender & $<0.001$ & & & \\
\hline Male & $2196(50.1)$ & $1194(54.4)$ & $1002(45.7)$ & \\
\hline Female & $2189(49.9)$ & $999(45.6)$ & $1190(54.3)$ & \\
\hline Age & 0.269 & & & \\
\hline Mean (SD) & $48.57(17.70)$ & $48.24(17.17)$ & $48.89(\mid 8.21)$ & 0.227 \\
\hline$<50$ years & $2314(52.8)$ & $1139(51.9)$ & $1175(53.6)$ & \\
\hline$\geq 50$ years & $2071(47.2)$ & $1054(48.1)$ & $1017(46.4)$ & \\
\hline Race & 0.581 & & & \\
\hline Non-Hispanic white & $2009(45.8)$ & $1014(46.2)$ & $995(45.4)$ & \\
\hline Non-Hispanic black & $887(20.2)$ & $438(20.0)$ & $449(20.5)$ & \\
\hline Mexican American & $649(14.8)$ & $314(14.3)$ & $335(15.3)$ & \\
\hline Other Hispanic & $449(10.2)$ & $219(10.0)$ & $230(10.5)$ & \\
\hline Other & $391(8.0)$ & $208(9.5)$ & $183(8.3)$ & \\
\hline Education & $<0.001$ & & & \\
\hline Less than high school & $1130(25.8)$ & $449(20.5)$ & $681(31.1)$ & \\
\hline High school or equivalent & $1010(23.0)$ & $466(21.2)$ & $544(24.8)$ & \\
\hline College or above & $2240(5 I .1)$ & $1275(58.1)$ & $965(44.0)$ & \\
\hline Other & $5(0.1)$ & $3(0.1)$ & $2(0.1)$ & \\
\hline Marital status & $<0.001$ & & & \\
\hline Married & $2275(51.9)$ & $1225(55.9)$ & $1050(47.9)$ & \\
\hline Unmarried & $2110(48.1)$ & $968(44.1)$ & $1142(52.1)$ & \\
\hline Family poverty ratio (\%) & $<0.001$ & & & \\
\hline$<1.3 \%$ & $146 \mid(33.3)$ & $619(28.2)$ & $842(38.4)$ & \\
\hline $1.3-3.5 \%$ & $1572(35.8)$ & $778(35.5)$ & 794 (36.2) & \\
\hline$\geq 3.5 \%$ & $1352(30.8)$ & $796(36.3)$ & $556(25.4)$ & \\
\hline BMI $\left(\mathrm{kg} / \mathrm{m}^{2}\right)$ & 0.091 & & & \\
\hline Mean (SD) & $28.98(6.79)$ & $28.86(6.75)$ & $29.09(6.83)$ & 0.288 \\
\hline$<25 \mathrm{~kg} / \mathrm{m}^{2}$ & $1319(30.1)$ & $634(28.9)$ & $685(31.2)$ & \\
\hline$\geq 25 \mathrm{~kg} / \mathrm{m}^{2}$ & $3066(69.9)$ & $1559(71.1)$ & $1507(68.8)$ & \\
\hline Hypertension & 0.983 & & & \\
\hline Yes & |49| (34.0) & $746(34.0)$ & $745(34.0)$ & \\
\hline No/Unknown & $2894(66.0)$ & $1447(66.0)$ & $1447(66.0)$ & \\
\hline Diabetes & 0.117 & & & \\
\hline Yes & $503(11.5)$ & $235(10.7)$ & $268(12.2)$ & \\
\hline No/Unknown & $3882(88.5)$ & $1958(89.3)$ & $1924(87.8)$ & \\
\hline Vigorous recreational activities & $<0.001$ & & & \\
\hline Yes & $941(21.5)$ & $563(25.7)$ & $378(17.2)$ & \\
\hline No & $3444(78.5)$ & $1630(74.3)$ & $1814(82.8)$ & \\
\hline Moderate recreational activities & $<0.001$ & & & \\
\hline Yes & $1740(39.7)$ & $964(44.0)$ & $776(35.4)$ & \\
\hline No & $2645(60.3)$ & $1229(56.0)$ & $1416(64.6)$ & \\
\hline Kidney stone & 0.023 & & & \\
\hline Yes & $376(8.6)$ & $167(7.6)$ & $209(9.5)$ & \\
\hline No & $4009(91.4)$ & $2026(92.4)$ & $1983(90.5)$ & \\
\hline
\end{tabular}

(Continued) 
Table 2 (Continued).

\begin{tabular}{|l|l|l|l|}
\hline \multirow{2}{*}{ Characteristic } & Total & \multicolumn{2}{|c|}{ Total PAHs Level } \\
\cline { 2 - 4 } & No. (\%) & Low Group & High Group \\
\hline Blood urea nitrogen, $\mathrm{mg} / \mathrm{dL}$ & $13.10(5.78)$ & $12.69(5.70)$ & $13.50(5.84)$ \\
\hline Creatinine, $\mathrm{mg} / \mathrm{dL}$ & $0.89(0.34)$ & $5.42(1.47)$ & $5.58(1.44)$ \\
\hline Uric acid, $\mathrm{mg} / \mathrm{dL}$ & $5.50(\mathrm{l}) .45)$ & $0.87(0.36)$ & $0.91(0.31)$ \\
\hline eGFR & $94.99(23.6)$ & $96.59(23.34)$ & $<0.001$ \\
\hline
\end{tabular}

Notes: ${ }^{a}$ For categorical variables, $\mathrm{P}$ values were analyzed by chi-square tests. For continuous variables, the $t$-test for slope was used in generalized linear models. Abbreviations: BMI, body mass index; PAHs, polycyclic aromatic hydrocarbons; eGFR, estimated glomerular filtration rate.

Table 3 Adjusted Odds Ratios for Associations Between the Polycyclic Aromatic Hydrocarbons and the Presence of Kidney Stone in NHANES 2007-2012 ${ }^{\text {a }}$

\begin{tabular}{|c|c|c|c|c|c|c|}
\hline \multirow[t]{2}{*}{ Polycyclic Aromatic Hydrocarbons } & \multicolumn{2}{|l|}{ Basic Model } & \multicolumn{2}{|l|}{ Core Model } & \multicolumn{2}{|l|}{ Extended Model } \\
\hline & aOR (95\% Cl) & $\mathbf{P}$ & aOR (95\% CI) & $\mathbf{P}$ & aOR (95\% Cl) & $\mathbf{P}$ \\
\hline \multicolumn{7}{|l|}{ Total PAHs } \\
\hline Low group & 1.00 & 1.00 & 1.00 & & & \\
\hline High group & $1.35(1.09-1.68)$ & 0.006 & $1.38(1.11-1.72)$ & 0.004 & $1.32(1.06-1.64)$ & 0.013 \\
\hline \multicolumn{7}{|l|}{ I-hydroxynaphthalene } \\
\hline Low group & 1.00 & 1.00 & 1.00 & & & \\
\hline High group & $1.13(0.91-1.40)$ & 0.272 & $1.12(0.90-1.39)$ & 0.317 & I.II (0.89-I.38) & 0.353 \\
\hline \multicolumn{7}{|l|}{ 2-hydroxynaphthalene } \\
\hline Low group & 1.00 & 1.00 & 1.00 & & & \\
\hline High group & 1.33 (1.07-I.65) & 0.009 & $1.41(1.13-1.75)$ & 0.003 & 1.37 (I.I0-I.7I) & 0.005 \\
\hline \multicolumn{7}{|l|}{ 3-hydroxyfluorene } \\
\hline Low group & 1.00 & 1.00 & 1.00 & & & \\
\hline High group & $1.18(0.95-1.46)$ & 0.131 & $1.19(0.95-1.48)$ & 0.123 & 1.18 (0.94-1.47) & 0.147 \\
\hline \multicolumn{7}{|l|}{ 2-hydroxyfluorene } \\
\hline Low group & 1.00 & 1.00 & 1.00 & & & \\
\hline High group & $1.25(1.01-1.55)$ & 0.039 & $1.27(1.02-1.58)$ & 0.033 & $1.25(1.00-1.55)$ & 0.051 \\
\hline \multicolumn{7}{|l|}{ 3-hydroxyphenanthrene } \\
\hline Low group & 1.00 & 1.00 & 1.00 & & & \\
\hline High group & $1.14(0.92-1.41)$ & 0.224 & $1.09(0.88-1.36)$ & 0.426 & $1.09(0.87-1.35)$ & 0.463 \\
\hline \multicolumn{7}{|l|}{ I-hydroxyphenanthrene } \\
\hline Low group & 1.00 & 1.00 & 1.00 & & & \\
\hline High group & $1.33(1.07-1.65)$ & 0.009 & $1.27(1.02-1.58)$ & 0.034 & $1.24(1.00-1.54)$ & 0.046 \\
\hline \multicolumn{7}{|l|}{ 2-hydroxyphenanthrene } \\
\hline Low group & 1.00 & 1.00 & 1.00 & & & \\
\hline High group & $1.32(1.07-1.64)$ & 0.011 & I. 17 (0.94-I.46) & 0.172 & I.I6 (0.93-I.44) & 0.200 \\
\hline \multicolumn{7}{|l|}{ I-hydroxypyrene } \\
\hline Low group & 1.00 & 1.00 & 1.00 & & & \\
\hline High group & I.2I (0.97-I.50) & 0.089 & $1.16(0.93-1.45)$ & 0.190 & $\mathrm{I} .15(0.92-\mathrm{I} .44)$ & 0.212 \\
\hline \multicolumn{7}{|l|}{ 9-hydroxyfluorene } \\
\hline Low group & 1.00 & 1.00 & 1.00 & & & \\
\hline High group & $1.41(1.14-1.75)$ & 0.002 & $1.38(1.11-1.72)$ & 0.004 & $1.36(1.09-1.70)$ & 0.007 \\
\hline
\end{tabular}

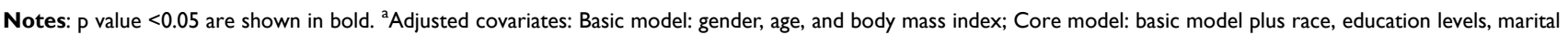
status and family poverty; Extended model: core model plus hypertension, diabetes, vigorous recreational activities, moderate recreational activities, blood urea nitrogen, uric acid, creatinine and estimated glomerular filtration rate (eGFR).

Abbreviations: $\mathrm{Cl}$, confidence interval; aOR, adjusted odds ratio. 
A

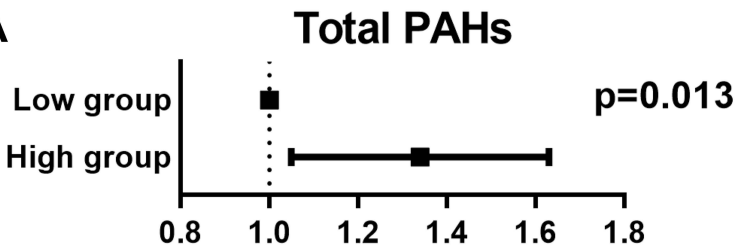

C

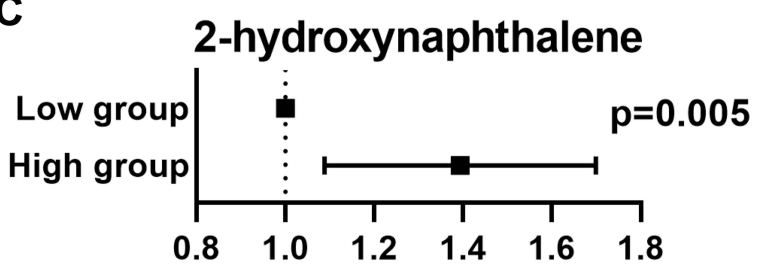

E

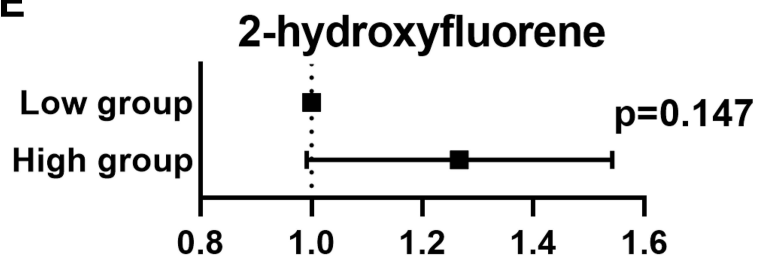

G

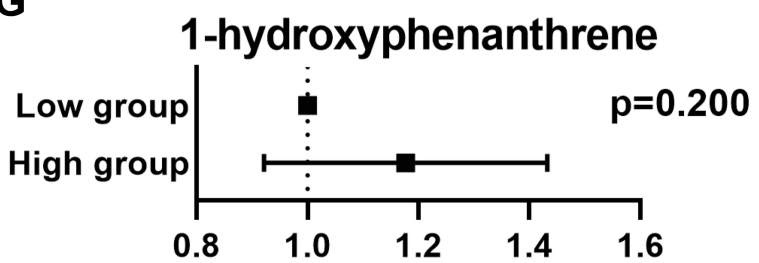

I

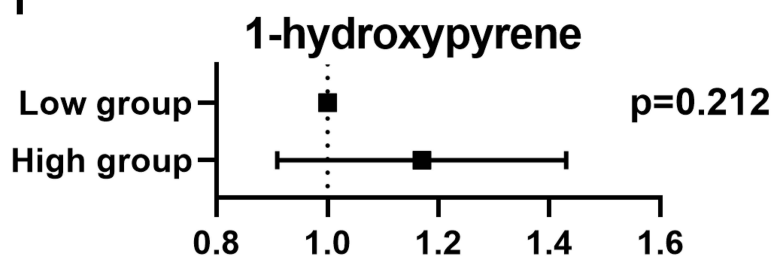

B 1-hydroxynaphthalene

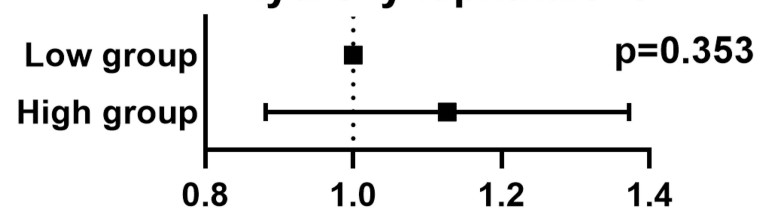

D

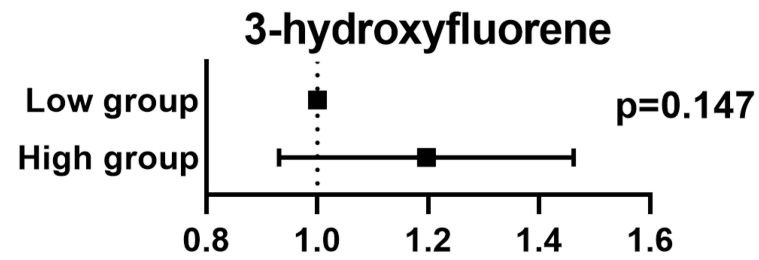

$\mathbf{F}$

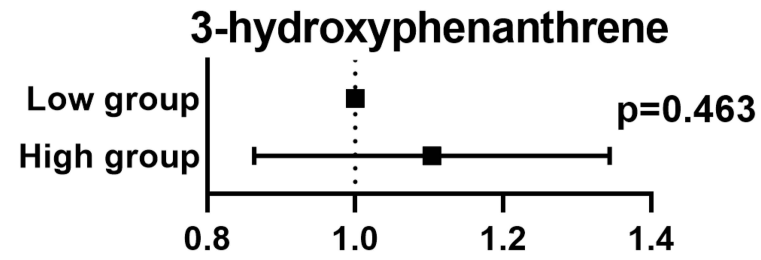

H

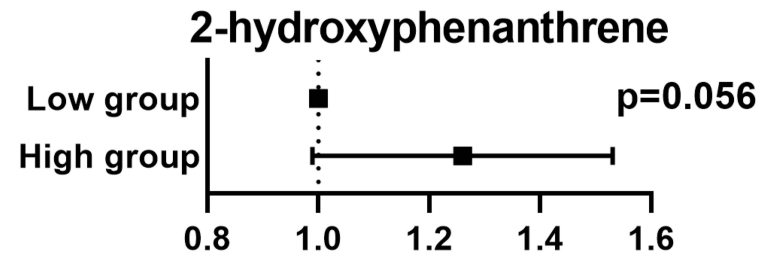

J

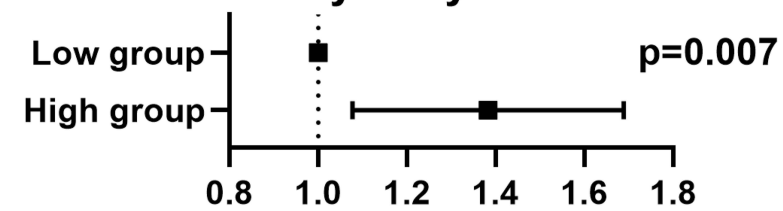

Figure 2 Adjusted odds ratios for associations between the PAHs and the presence of kidney stone in NHANES 2007-20I2. (A) Total PAHs; (B) I-hydroxynaphthalene; (C) 2-hydroxynaphthalene; (D) 3-hydroxyfluorene; (E) 2-hydroxyfluorene; (F) 3-hydroxyphenanthrene; (G) I-hydroxyphenanthrene; (H) 2-hydroxyphenanthrene; (I) I-hydroxypyrene; (J) 9-hydroxyfluorene.

the occurrence risk of bladder cancer. ${ }^{24}$ Polycyclic aromatic hydrocarbon metabolites may lead to insulin resistance and b-cell dysfunction. ${ }^{25}$ Participants with higher total PAHs were concentrated in female, college or above, unmarried, family poverty ratio $<1.3 \%$, less recreational activities, kidney stones, higher creatinine, higher blood urea nitrogen, higher uric acid and lower eGFR. Li et al reported that PAHs exposure may be associated with albuminuria. ${ }^{26}$ PAHs may cause kidney damage by exacerbating kidney dysfunction. Polycyclic aromatic hydrocarbon metabolites can induce oxidative stress, even cause DNA damage in cells, and promote cardiovascular and cerebrovascular diseases. ${ }^{27}$ PAHs are absorbed by humans mainly through the respiratory tract, digestive tract, skin, and some will be excreted in urine or feces after being metabolized. ${ }^{28,29}$ Some of them further covalently bind with DNA or proteins, thus destroying the structure and function of proteins and nucleic acids, breaking the oxidation-antioxidant balance in the body. ${ }^{30}$ Only a few unmetabolized PAH prototypes will be eliminated from the body through urine or bile and feces. ${ }^{31}$ Studying the relationship between PAH metabolites and related diseases can more effectively explain the pathogenic mechanism. 


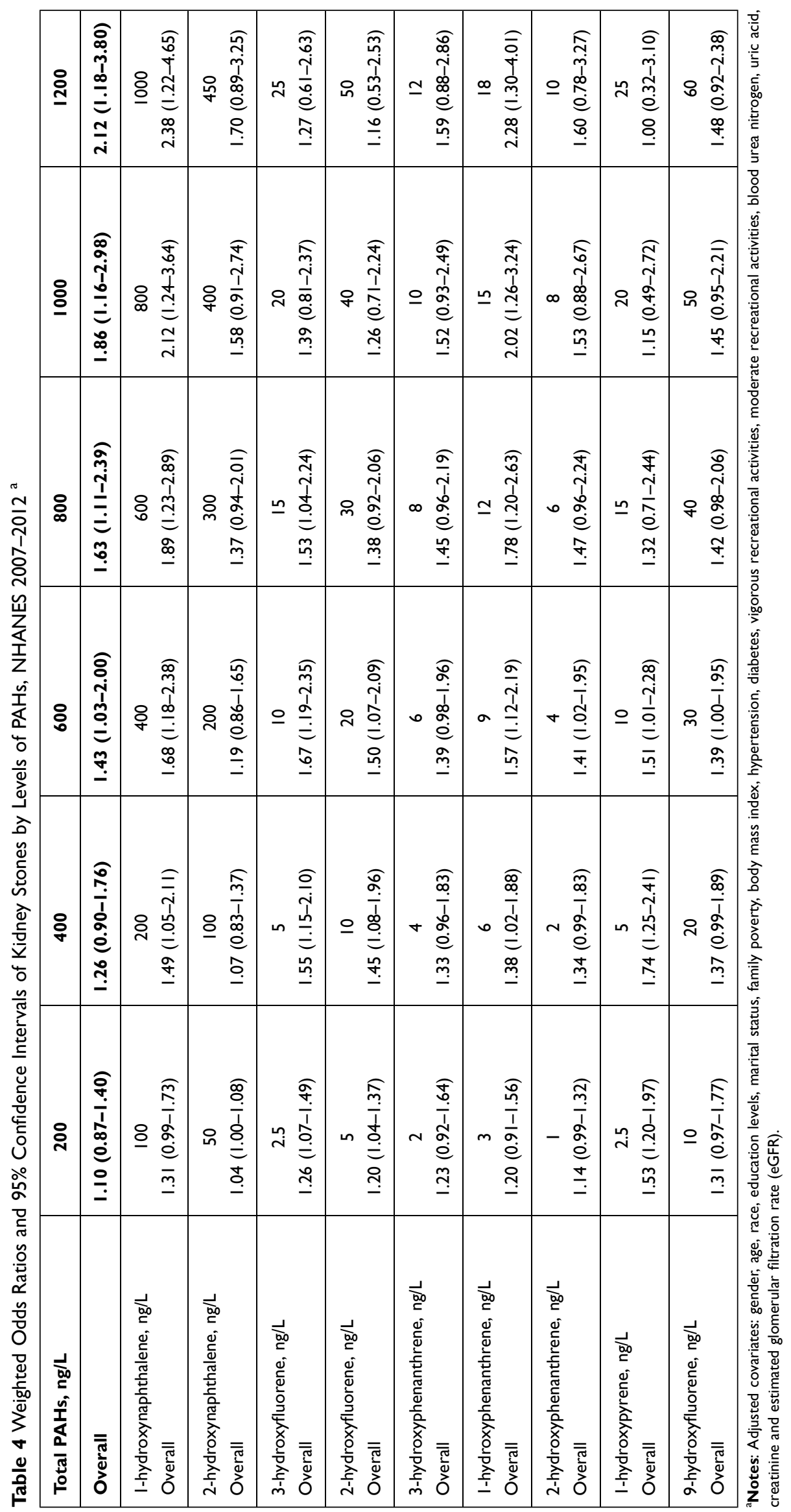



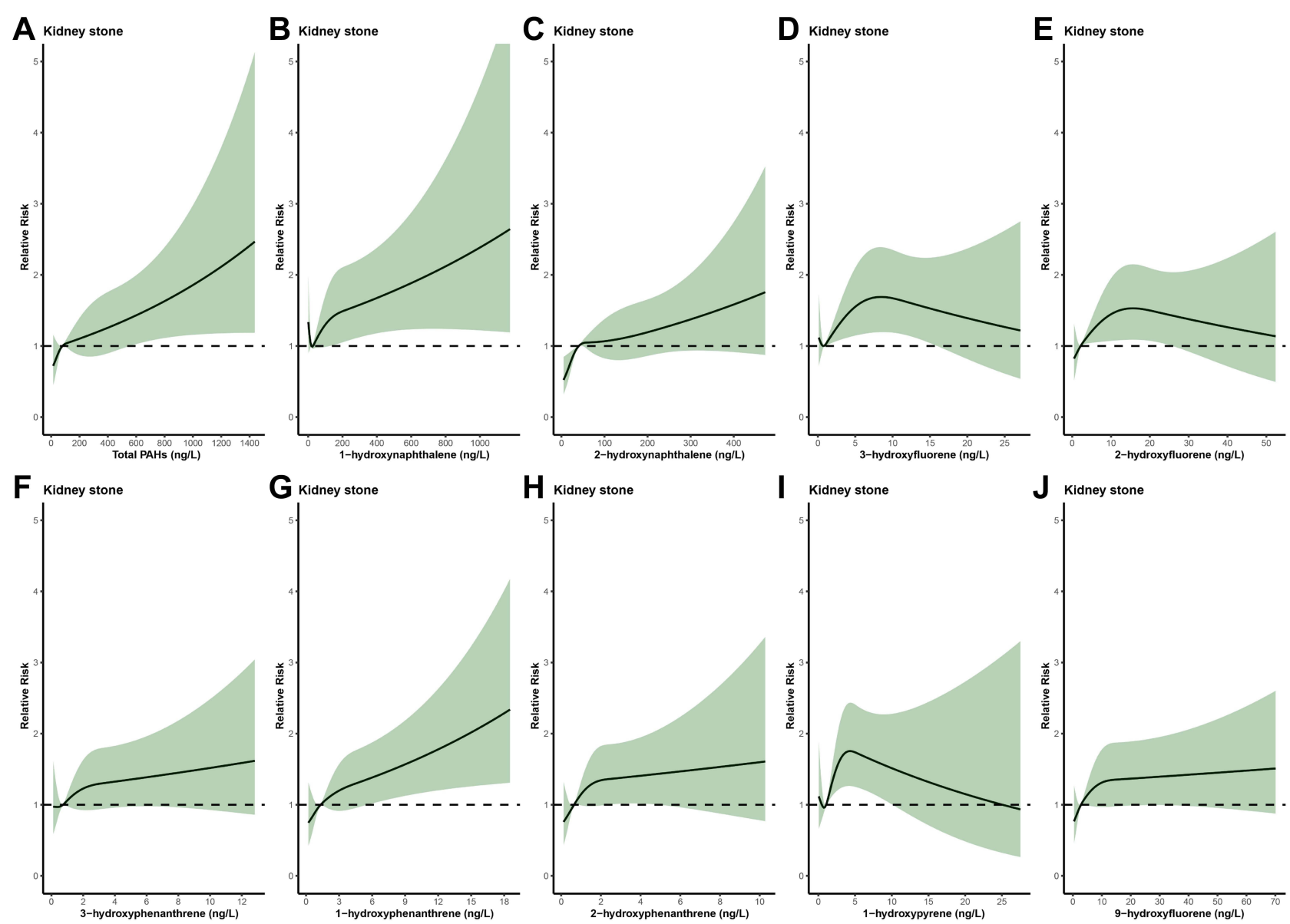

3-hydroxyphenanthrene (ng/l)

2-hydroxyphenanthrene (ng/L)

1-hydroxypyrene (ng/L)

9-hydroxyfluorene (ng/

Figure 3 Relative risk for kidney stones based on PAHs level. The solid black lines represent aORs according to restricted cubic splines for PAHs level. The shaded areas represent upper and lower $95 \% \mathrm{Cls}$. Adjustment factors are as same as which presented in extended model of Table 3. (A) Total PAHs; (B) I-hydroxynaphthalene; (C) 2-hydroxynaphthalene; (D) 3-hydroxyfluorene; (E) 2-hydroxyfluorene; (F) 3-hydroxyphenanthrene; (G) I-hydroxyphenanthrene; (H) 2-hydroxyphenanthrene; (I) I-hydroxypyrene; (J) 9-hydroxyfluorene.

At present, more and more studies on the occurrence of kidney stones are related to oxidative stress and inflammation. ${ }^{32}$ Oxidative stress is defined as the imbalance between oxidants and antioxidants. Reactive oxygen species (ROS) can mediate oxidative stress in mitochondria. At the same time, reactive oxygen species participate in $\mathrm{CaOx}$ crystals to promote renal tubular epithelial cell damage and apoptosis. Apoptosis and cell membrane structure changes can cause crystal adhesion and finally initiate a cascade of kidney stones. ${ }^{33,34}$ Huang's research showed that lipid peroxidation was related to renal tubular epithelial cell damage, which was related to free radicals produced by patients with $\mathrm{CaOx}$ stones. ${ }^{35} \mathrm{PAHs}$ can produce a large amount of ROS in the metabolic process of the human body. When it exceeds the body's compensation, the excessive ROS can attack the biological macromolecules, causing oxidative stress, inflammatory response and immune response. Vijaya et al found that
PAHs activate the oxidative stress mechanism after exposure, and the active molecules generated in the reaction can activate multiple signaling pathways in cells closely related to the immune cell function. ${ }^{36}$ Our study provides potential results for studying the relationship between PAHs exposure and the prevalence of kidney stones. The metabolism of PAHs may increase the incidence of kidney stones by increasing oxidative stress.

Previous studies have also examined the association between PAHs and celiac disease and kidney stones in adults. By collecting information from 5,560 adult participants in NHANES 2011-2012, Shiue found that after adjusting for urinary creatinine, age, sex, BMI, household income to poverty ratio, serum cotinine, alcohol consumption habits, education level, and physical activity level, urinary 2-hydroxyfluorene, 3-hydroxyfluorene, 1-hydroxyphenanthrene, 1-hydroxypyrene, and 2-hydroxynaphthalene were associated with kidney stones. ${ }^{37}$ In our study, 
we expanded the years of data collection and adjusted for sex, age, race, education level, marital status, household income to poverty ratio, BMI, hypertension, diabetes, physical activity level (vigorous and moderate), blood urea nitrogen, uric acid, creatinine, and eGFR. We found that total PAHs, 2-hydroxynaphthalene, 1-hydroxyphenanthrene, 2-hydroxyphenanthrene, and 9-hydroxy fluorene were significantly and positively correlated with the risk of kidney stones. Compared with the low group, the high group showed significant associations in total PAHs [OR (95\% CI), 1.32 (1.06-1.64)], 2-hydroxynaphthalene [OR (95\% CI), 1.37 (1.10-1.71)], 1-hydroxyphenanthrene [OR (95\% CI), 1.24 (1.00-1.54)] and 9-hydroxyfluorene [OR (95\% CI), 1.36 (1.09-1.70)]. In addition, we constructed dose-response curves between each PAHs and kidney stones to more visually demonstrate the risk relationship between each PAHs and kidney stones. Furthermore, we found that stone formers had higher blood urea nitrogen, creatinine, uric acid, total PAHs and lower eGFR than nonstone formers, possibly because PAHs may cause kidney damage by exacerbating kidney dysfunction, which in turn affects the excretion of PAHs. ${ }^{37,38}$

Using data from a large sample of NHANES in the United States, we can explore the relationship between PAHs and the prevalence of kidney stones while adjusting potential variables. Later, we can further discover its toxicity, pathogenic mechanism, and metabolic pathways, which will provide new methods for preventing and treating related diseases. There were several limitations of this study. First, due to the cross-sectional nature of the study, we could not fully determine the relationship between $\mathrm{PAH}$ exposure and kidney stones, which requires further prospective studies to verify its accuracy. Second, there is no information about the type of kidney stones. In addition, data on kidney stones were based on self-reporting by participants rather than professional diagnosis by physicians.

\section{Conclusions}

Our study shows that High levels of total PAHs were positively associated with the risk of kidney stones in the US population. Longitudinal studies are still needed to determine the exact relationship between PAHs and kidney stones.

\section{Data Sharing Statement}

The datasets used and analyzed during the current study are available from Ming Chen on reasonable request.

\section{Acknowledgments}

The authors thank all patients and investigators involved in these studies.

\section{Funding}

This work was supported by grants from Jiangsu Provincial Medical Youth Talent (QRNC2016820), National Natural Science Foundation of China (NO. 81670632 and 81900618), and the Scientific Research Foundation of Graduate School of Southeast University (YBPY2173).

\section{Disclosure}

The authors report no conflicts of interest in this work.

\section{References}

1. Khan SR, Canales BK, Dominguez-Gutierrez PR. Randall's plaque and calcium oxalate stone formation: role for immunity and inflammation. Nat Rev Nephrol. 2021.

2. Khan SR, Pearle MS, Robertson WG, et al. Kidney stones. Nat Rev Dis Primers. 2016;2:16008.

3. Coe FL, Worcester EM, Evan AP. Idiopathic hypercalciuria and formation of calcium renal stones. Nat Rev Nephrol. 2016;12 (9):519-533.

4. Turk C, Petrik A, Sarica K, et al. EAU guidelines on interventional treatment for urolithiasis. Eur Urol. 2016;69(3):475-482.

5. Ormanji MS, Rodrigues FG, Heilberg IP. Dietary recommendations for bariatric patients to prevent kidney stone formation. Nutrients. 2020;12:5

6. Ona-Ruales JO, Ruiz-Morales Y, Wise SA. Identification and quantification of seven fused aromatic rings $\mathrm{C} 26 \mathrm{H} 14$ peri-condensed benzenoid polycyclic aromatic hydrocarbons in a complex mixture of polycyclic aromatic hydrocarbons from coal tar. J Chromatogr A. 2016;1442:83-93.

7. Hu Z, Li Y, Yang Y, et al. Serum lipids mediate the relationship of multiple polyaromatic hydrocarbons on non-alcoholic fatty liver disease: a population-based study. Sci Total Environ. 2021;780:146563.

8. Cave MR, Wragg J, Beriro DJ, et al. An overview of research and development themes in the measurement and occurrences of polyaromatic hydrocarbons in dusts and particulates. J Hazard Mater. 2018;360:373-390.

9. Teixeira EC, Agudelo-Castaneda DM, Mattiuzi CD. Contribution of polycyclic aromatic hydrocarbon $(\mathrm{PAH})$ sources to the urban environment: a comparison of receptor models. Sci Total Environ. 2015;538:212-219.

10. Wurl O, Obbard JP. A review of pollutants in the sea-surface microlayer (SML): a unique habitat for marine organisms. Mar Pollut Bull. 2004;48(11-12):1016-1030.

11. Liu G, Niu Z, Van Niekerk D, Xue J, Zheng L. Polycyclic aromatic hydrocarbons (PAHs) from coal combustion: emissions, analysis, and toxicology. Rev Environ Contam Toxicol. 2008;192:1-28.

12. Dugheri S, Bonari A, Gentili M, et al. High-throughput analysis of selected urinary hydroxy polycyclic aromatic hydrocarbons by an innovative automated solid-phase microextraction. Molecules. 2018;23:8.

13. Mumtaz MM, George JD, Gold KW, Cibulas W, DeRosa CT. ATSDR evaluation of health effects of chemicals IV Polycyclic Aromatic Hydrocarbons (PAHs): understanding a complex problem. Toxicol Ind Health. 1996;12(6):742-971. 
14. Rosario Filho NA, Urrutia-Pereira M, D'Amato G, et al. Air pollution and indoor settings. World Allergy Organ J. 2021;14(1):100499.

15. Moller P, Scholten RH, Roursgaard M, Krais AM. Inflammation, oxidative stress and genotoxicity responses to biodiesel emissions in cultured mammalian cells and animals. Crit Rev Toxicol. 2020;50 (5):383-401.

16. Vogel CFA, Van Winkle LS, Esser C, Haarmann-Stemmann T. The aryl hydrocarbon receptor as a target of environmental stressors Implications for pollution mediated stress and inflammatory responses. Redox Biol. 2020;34:101530.

17. Mao W, Hu Q, Chen S, et al. Polyfluoroalkyl chemicals and the risk of kidney stones in US adults: a population-based study. Ecotoxicol Environ Saf. 2021;208:111497.

18. Desquilbet L, Mariotti F. Dose-response analyses using restricted cubic spline functions in public health research. Stat Med. 2010;29 (9):1037-1057.

19. Sfoungaristos S, Gofrit ON, Yutkin V, Pode D, Duvdevani M. Prevention of renal stone disease recurrence. A systematic review of contemporary pharmaceutical options. Expert Opin Pharmacother. 2015;16(8):1209-1218.

20. Min YS, Lim HS, Kim H. Biomarkers for polycyclic aromatic hydrocarbons and serum liver enzymes. Am J Ind Med. 2015;58 (7):764-772

21. Parvez F, Lauer FT, Factor-Litvak P, et al. Assessment of arsenic and polycyclic aromatic hydrocarbon (PAH) exposures on immune function among males in Bangladesh. PLoS One. 2019;14(5): e0216662.

22. Bosetti C, Boffetta P, La Vecchia C. Occupational exposures to polycyclic aromatic hydrocarbons, and respiratory and urinary tract cancers: a quantitative review to 2005. Ann Oncol. 2007;18 (3):431-446

23. Smiljevska-Ristovska V, Sabriu-Haxhijaha A, Ristoski T, Kosharkoska-Spasovska F, Krstanoski L, Dimitrova-Shumkovska J. Markers involved in proinflammatory effects by environmental toxicants. Toxicol Mech Methods. 2020;30(8):570-579.

24. Mallin K. A nested case-control study of bladder cancer incidence in a steel manufacturing plant. Am $J$ Ind Med. 1998;34 (4):393-398.

25. Yang L, Zhou Y, Sun H, et al. Dose-response relationship between polycyclic aromatic hydrocarbon metabolites and risk of diabetes in the general Chinese population. Environ Pollut. 2014;195:24-30.

26. Li J, Fan H, Liu K, et al. Associations of urinary polycyclic aromatic hydrocarbons with albuminuria in U.S. adults, NHANES 2003-2014. Ecotoxicol Environ Saf. 2020;195:110445.
27. Palackal NT, Lee SH, Harvey RG, Blair IA, Penning TM. Activation of polycyclic aromatic hydrocarbon trans-dihydrodiol proximate carcinogens by human aldo-keto reductase (AKR1C) enzymes and their functional overexpression in human lung carcinoma (A549) cells. J Biol Chem. 2002;277(27):24799-24808.

28. Ramesh A, Walker SA, Hood DB, Guillen MD, Schneider K, Weyand EH. Bioavailability and risk assessment of orally ingested polycyclic aromatic hydrocarbons. Int $J$ Toxicol. 2004;23 (5):301-333.

29. Campo L, Addario L, Buratti M, et al. Biological monitoring of exposure to polycyclic aromatic hydrocarbons by determination of unmetabolized compounds in urine. Toxicol Lett. 2006;162(23):132-138.

30. Brucker N, Moro AM, Charao MF, et al. Biomarkers of occupational exposure to air pollution, inflammation and oxidative damage in taxi drivers. Sci Total Environ. 2013;463-464:884-893.

31. Strickland P, Urinary KD. 1-hydroxypyrene and other PAH metabolites as biomarkers of exposure to environmental PAH in air particulate matter. Toxicol Lett. 1999;108(2-3):191-199.

32. de Water R, Leenen PJ, Noordermeer C, et al. Cytokine production induced by binding and processing of calcium oxalate crystals in cultured macrophages. Am J Kidney Dis. 2001;38(2):331-338.

33. Thamilselvan V, Menon M, Thamilselvan S. Oxalate at physiological urine concentrations induces oxidative injury in renal epithelial cells: effect of alpha-tocopherol and ascorbic acid. BJU Int. 2014;114 (1): $140-150$.

34. Kizivat T, Smolic M, Maric I, et al. Antioxidant pre-treatment reduces the toxic effects of oxalate on renal epithelial cells in a cell culture model of urolithiasis. Int J Environ Res Public Health. $2017 ; 14: 1$

35. Huang HS, Ma MC, Chen CF, Chen J. Lipid peroxidation and its correlations with urinary levels of oxalate, citric acid, and osteopontin in patients with renal calcium oxalate stones. Urology. 2003;62 (6):1123-1128.

36. Vijaya Padma V, Kalai Selvi P, Sravani S. Protective effect of ellagic acid against TCDD-induced renal oxidative stress: modulation of CYP1A1 activity and antioxidant defense mechanisms. Mol Biol Rep. 2014;41(7):4223-4232.

37. Shiue I. Urinary polyaromatic hydrocarbons are associated with adult celiac disease and kidney stones: USA NHANES, 2011-2012. Environ Sci Pollut Res Int. 2016;23(4):3971-3977.

38. Krajka-Kuzniak V, Baer-Dubowska W. The effects of tannic acid on cytochrome P450 and Phase II enzymes in mouse liver and kidney. Toxicol Lett. 2003;143(2):209-216.
International Journal of General Medicine

\section{Publish your work in this journal}

The International Journal of General Medicine is an international, peer-reviewed open-access journal that focuses on general and internal medicine, pathogenesis, epidemiology, diagnosis, monitoring and treatment protocols. The journal is characterized by the rapid reporting of reviews, original research and clinical studies across all disease areas. The manuscript management system is completely online and includes a very quick and fair peer-review system, which is all easy to use. Visit http://www.dovepress.com/ testimonials.php to read real quotes from published authors. 